\title{
Prediction of Curling Distance of Dry-Relaxed Cotton Plain Knitted Fabrics
}

Part 2 : Experimental Study and Regression Analysis for Prediction of Curling Distance

\author{
Nuray Ucar* \\ Istanbul Technical University, Faculty of Mechanical, Textile Engineering Department, Taksim, Istanbul, TURKEY \\ *Current Address: Georgia Institute of Technology, 801 Ferst Drive, School of Textile \& Fiber Engineering, \\ Atlanta, Georgia, 30332-0295, USA
}

Received 9 December 1999 ; accepted for publication 29 September 2000

\begin{abstract}
As mentioned in the Part 1 [ 9 ], many studies on the curling tendency of knitted fabrics have been done. However, until now, it was not possible to predict the exact curling distance of a fabric. Curling distance is very important in the garment industry where the glued-edge of a fabric is thought of as a material loss. Thus, the aim of present study is to determine the curling distance of fabrics before production. During this study, multiple regression analysis was done between fabric parameters that are know before production and curling distances that are measured by experimentally. In past experimental studies, the curling tendency has been measured because of the difficulties in measuring the curling distance. Thus, in this paper, a new method has been developed to measure the curling distance. The following results have been obtained:

(1) During the regression analysis, $r^{2}(r$ : coefficients of correlation) have been found to be 0.75 for both side curling and top/bottom curling ( $\mathrm{C}_{\mathrm{s}}$-side curling, $\mathrm{C}_{\mathrm{tb}}$-top/bottom curling, Equation 1 and 2 , respectively). Thus, it is possible to predict the curling distance before production, with in a reasonable approximation, using know fabric and yarn parameters. These parameters are loop length [7] that is determined before production, yarn count to calculate yarn diameter and bending rigidity of the yarn. These properties are know before production by experimental, calculation or by definition.

(2) As pointed out in Part 1 [9] and seen from Table 2 : the values of the moments about the $x$ axis (about an axis parallel to the courses) are higher than the values of the moments about the $y$ axis (about an axis parallel to the wales) $\left(\mathrm{M}_{\mathrm{x}}\right.$ is bigger than $\mathrm{M}_{\mathrm{y}}$ ). But again as seen from Table 2 ; curling distance about the $\mathrm{x}$ axis $\left(\mathrm{C}_{\mathrm{tb}}\right.$-top/bottom curling) is lower then curling distance about the $\mathrm{y}$ axis $\left(\mathrm{C}_{5}\right.$-side curling), since bending rigidity of the fabric for bending about the $\mathrm{x}$ axis (about an axis parallel to the courses) is higher than the bending rigidity of the fabric about the y axis [ $3-5$ ].
\end{abstract}

Original article has been published in Journal of Textile Engineering, Vol. 46. No. 4 (2000) 